\title{
Update on the subcutaneous administration of rituximab in Canadian cancer centres
}

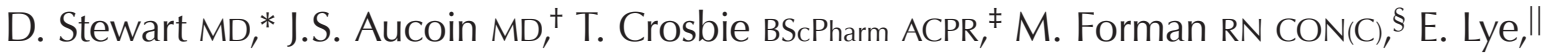

A. Christofides MSc RD, ${ }^{\#}$ and A. Mitha RPh BSc Phm**

\begin{abstract}
Results of studies comparing subcutaneous (SC) with intravenous (IV) rituximab indicate that the two formulations are comparable in efficacy, but most patients and health care professionals prefer the sc route, commonly because of shorter chair time and reduced risk of infusion-related reactions. Recent Canadian data, including those from the SCuBA study reported here, support the results of earlier international studies showing a reduction in preparation and administration time with the Sc formulation, lower cost of administration, and reduced drug wastage because of the fixed SC dosing. Given the significant time and cost savings of the SC formulation, that formulation is generally preferred over the IV formulation for the treatment of follicular lymphoma, diffuse large B cell lymphoma, and chronic lymphocytic leukemia.
\end{abstract}

Key Words Rituximab, subcutaneous administration

Curr Oncol. 2020 April:27(2)113-116

www.current-oncology.com

\section{BACKGROUND}

Rituximab is widely used for the treatment of B cell non-Hodgkin lymphoma, being a key component of most therapeutic regimens ${ }^{1-4}$. The $375 \mathrm{mg} / \mathrm{m}^{2}$ intravenous (IV) formulation involves dose calculations, infusion preparation, a long infusion duration, and titration of the infusion rate according to tolerability ${ }^{5}$. Complications of IV administration can include the risk of infusion-related reactions, which might result in a burden on health care resources and could impair the patient's quality of life. To provide a more convenient administration method, a fixed subcutaneous (SC) dose of rituximab $1400 \mathrm{mg}$ was developed.

Study results in non-Hodgkin lymphoma indicate that, compared with the $375 \mathrm{mg} / \mathrm{m}^{2}$ IV formulation, the $1400 \mathrm{mg}$ SC formulation is noninferior in pharmacokinetics and is associated with comparable response rates ${ }^{6-12}$. Moreover, the sc formulation is preferred by patients and health care providers, and reduces administration and chair time. Additional advantages include a reduced potential for dosing errors and drug wastage because of the fixed dose, reduced preparation time, and fewer infusion-related reactions.

The sc formulation of rituximab was approved for use in follicular lymphoma (FL) and diffuse large B cell lymphoma (DLBCL) by the European Commission in March $2014^{13}$ and by Health Canada in September $2016^{14}$. The approvals for FL and
DLBCL were based on results of the phase III SABRINA study, which demonstrated pharmacokinetic noninferiority of the sc compared with the IV formulation, with a similar efficacy and safety profile $\mathrm{e}^{10,15}$. In 2018, the $1600 \mathrm{mg}$ sC formulation was approved in Canada for the treatment of chronic lymphocytic leukemia based on data from the phase IB SAWYER study that also demonstrated pharmacokinetic noninferiority of the $1600 \mathrm{mg}$ sc formulation compared with the $500 \mathrm{mg} / \mathrm{m}^{2}$ IV formulation, with a comparable efficacy and safety profile ${ }^{12,16}$. The SC formulation of rituximab is currently funded across Canada for the treatment of FL, DLBCL, and chronic lymphocytic leukemia; the only exception is Quebec which, at the time of writing, funds it only for $\mathrm{FL}^{17}$. Although, to date, approximately $55 \%$ of patients receive the SC formulation, rates of conversion to SC from IV are lower in Quebec at approximately 6\% and in Saskatchewan at approximately 22\% (Hoffman-La Roche. Conversion rate from IV to SC rituximab by province. Data on file, 2019).

Since its approval, the Sc formulation has been increasingly used in Canadian practice, providing first-hand experience for oncologists, pharmacists, and nurses. In addition, Canadian-specific data quantifying cost and time savings relating to the use of the Sc formulation in place of the IV formulation in Canadian systemic therapy suites have now been published ${ }^{14}$. The present study took a Canadian health care system perspective to model the 
effect of implementing SC rituximab in chemoimmunotherapy for FL and DLBCL. The model was most sensitive to sc market uptake, number of induction therapy cycles, and eligible patients. More than 3 years after the implementation of SC rituximab, it was estimated that 5762 Canadians would be receiving tha formulation, resulting in savings of 128,715 hours in systemic therapy suite time and approximately $\$ 40$ million in drug and administration costs. The estimated incremental savings for 1 full course of treatment when changing from IV to SC rituximab would amount to $\$ 5,017$ (costs) and 15.28 hours (preparation and administration) per DLBCL patient and to $\$ 12,212$ and 38.8 hours per FL patient.

The scuBA (Subcutaneous Benefit Analysis) study provides additional insight into the institutional, health care practitioner, and patient impacts of the administration differences between the two rituximab formulations. Results from a French version of the study conducted in 36 cancer centres across France were published in July $2018^{18}$. In the French study, results showed a mean chair time reduction of $73.8 \%$ and a corresponding gain in annual earnings of $€ 111,388$ with the sc formulation compared with the IV formulation of rituximab. The purpose of the present article is to report data from the scuBA study and to provide perspectives about the practical experience with the sc formulation of rituximab from Canadian oncologists, nurses, pharmacists, and patient advocacy groups.

\section{METHODS}

The SCuBA study was designed to evaluate the institutional, health care practitioner, and patient effects of SC or IV administration of rituximab. It included an online survey sent to a list of Canadian health care practitioners based at cancer centres known to be using the sc formulation of rituximab. The survey included questions about the current use of SC rituximab, pharmacy preparation time and cost, patient chair time, nurse administration time, and drug wastage with the two formulations.

\section{RESULTS}

Of the 55 participants from 25 cancer centres across Canada who completed the online survey, most came from Ontario ( $n=30$, Table I). Participants included physicians $(n=16)$, nurses $(n=23)$, and pharmacists $(n=16)$. Most participants reported their centres as being "full to capacity" ( $n=22$ of 41 ), with the remainder being "busy but manageable" $(n=19$ of 41$)$.

The average rituximab preparation time was greater with the IV than with the SC formulation [IV: $20.3 \mathrm{~min}-$ utes (range: 7-60 minutes); sc: 13.4 minutes (range: 2-31 minutes); Table II]. Participants reported an average drug preparation cost reduction of $33.5 \%$ with the sc formulation compared with the IV formulation. Similarly, the average nurse administration time was greater with the IV formulation [IV: 118.5 minutes (range: 19-390 minutes); sc: 32.2 minutes (range: 5-145 minutes)]. Likewise, the average chair time was greater with the IV formulation [IV: 166.9 minutes (range: 20-480 minutes); sc: 41.3; range: 10-110 minutes)]. Participants also reported an average reduction in drug wastage of $62.0 \%$ with the sc formulation (range: $20 \%-90 \%)$.

\section{Canadian Perspective}

The study by Stewart et al. ${ }^{14}$ about the impact of the sc formulation of rituximab on Canadian systemic therapy suites confirms the significant chair time, nursing time, and cost savings for SC administration compared with IV administration reported from other countries ${ }^{6-12}$. The scuBA study supports the foregoing findings and provides unique data from Canadian cancer centres reflecting real-world practice. Despite the limitations of a retrospective design, the scuBA data provide valuable insight for Canadian centres into the duration of preparation and administration of the sc formulation to aid in adjusting workflow and practices.

The fact that most Canadian systemic therapy suites are at capacity underscores the need for time- and costreduction strategies. A reduction in nursing administration and pharmacy preparation time allows staff to perform other duties, increasing efficiency in cancer care delivery. However, to benefit from the shortened treatment duration, workflow adjustments such that products are prepared in a timely manner might be required.

The dose of the SC rituximab formulation remains the same, regardless of patient body weight, resulting in less wastage because no vials need be discarded ${ }^{1}$. In addition, given that the Sc formulation in a syringe is stable for 48 hours, it can be refrigerated and used the next day if needed, which is especially useful for centres with higher patient volumes. In addition, fewer consumables are used in administering the sc formulation because the bags of saline, tubing, and IV pumps associated with the IV formulation are not required.

Although the occasional patient has a needle phobia or experiences an unacceptable injection site reaction, few patients make the switch back to IV from sc rituximab in the experience of the authors. For most patients, the benefits of the sc formulation are significant.

Diphenhydramine is often used as a pre-medication with IV rituximab. However, non-sedating antihistamines

TABLE I Demographics of SCuBA participants

\begin{tabular}{lcc}
\hline \multicolumn{1}{c}{ Variable } & \multicolumn{2}{c}{ Responders (n) } \\
\cline { 2 - 3 } & Total & Distribution \\
\hline HCP type & 55 & 23 \\
Nurse & & 16 \\
Pharmacist & & 16 \\
Physician & 55 & \\
Province & & 30 \\
Ontario & & 6 \\
New Brunswick & & 2 \\
Manitoba & & 10 \\
British Columbia & & 7 \\
Quebec & 41 & \\
Capacity of centre & & 19 \\
Full to capacity & & \\
Busy, but manageable & & \\
\hline
\end{tabular}

SCuBA = Subcutaneous Benefit Analysis study; $\mathrm{HCP}=$ health care provider. 
such as loratadine can be chosen as a pre-medication with the SC route. Avoiding at least 90 minutes with an IV and avoidance of daytime sedation can improve patient comfort, convenience, and time to accommodate other aspects of life. Moreover, the low risk of injection site reactions with SC administration is likely to be outweighed by the reduction in the risk of infusion-related reactions from IV administration. Patients given the sc formulation have uninterrupted nurse time, because sc administration allows the nurse to sit with the patient compared with the nurse coming and going during IV administration. Other potential advantages for caregivers include reduced waiting time and parking costs.

Some centres might feel less comfortable giving the SC formulation to patients with curable lymphomas such as

TABLE II Key SCuBA results

\begin{tabular}{|c|c|c|}
\hline \multirow[t]{2}{*}{ Variable } & \multicolumn{2}{|c|}{ Responders } \\
\hline & Total & Distribution \\
\hline $\begin{array}{l}\text { Indication for subcutaneous } \\
\text { formulation }(n)\end{array}$ & 39 & \\
\hline Diffuse large B cell lymphoma & & 36 \\
\hline Follicular lymphoma & & 37 \\
\hline Chronic lymphocytic leukemia & & 17 \\
\hline \multicolumn{3}{|l|}{ Pharmacy preparation time (minutes) } \\
\hline Intravenous & 44 & \\
\hline Average & & 20.3 \\
\hline Range & & $7-60$ \\
\hline Subcutaneous & 33 & \\
\hline Average & & 13.4 \\
\hline Range & & $2-31$ \\
\hline \multicolumn{3}{|l|}{ Preparation cost change $(\%)$} \\
\hline Reduction & 31 & \\
\hline Average & & 33.5 \\
\hline Range & & $5-80$ \\
\hline Increase & 1 & 0.5 \\
\hline \multicolumn{3}{|l|}{ Average chair time (minutes) } \\
\hline Intravenous & 41 & \\
\hline Average & & 166.9 \\
\hline Range & & $20-480$ \\
\hline Subcutaneous & 39 & \\
\hline Average & & 41.3 \\
\hline Range & & $10-110$ \\
\hline
\end{tabular}

Nurse administration time (minutes)

\begin{tabular}{|c|c|c|}
\hline Intravenous & 40 & \\
\hline Average & & 118.5 \\
\hline Range & & $19-390$ \\
\hline Subcutaneous & 39 & \\
\hline Average & & 32.2 \\
\hline Range & & $5-145$ \\
\hline
\end{tabular}

Wastage $(\%)$

Reduction with subcutaneous

23

Average

62.0

Range

20-90

Increase with subcutaneous

0

SCuBA = Subcutaneous Benefit Analysis study.

DLBCL, because they do not want to jeopardize any chance of a cure. However, there is no evidence or biologic rationale to support that concern ${ }^{10,12,15,16}$. Moreover, where patients are currently being given the IV formulation, it might be reasonable to switch to the sc formulation for the remainder of therapy, given the results of the studies already cited.

For centres with large patient volumes or for small centres with limited resources, the cost and time benefits of the sc formulation are particularly notable when the treatment regimen has no other IV component-for example, maintenance rituximab. It is also important to note that, with the advent of rituximab biosimilars, a less costly IV formulation could be available in the future. At that time, the benefits of adopting SC rituximab in terms of time savings will have to be weighed against the cost of the biosimilars. If the cost for SC rituximab and for an IV rituximab biosimilar were to be equal, patients and health care practitioners will probably prefer to keep the benefits offered by the sc route.

\section{CONCLUSIONS}

Most Canadian systemic therapy suites are full to capacity. The comparable efficacy, significant time and cost savings, and preference for the SC over the IV formulation of rituximab suggest that, to increase efficiency in cancer care delivery, cancer centres should consider SC administration for most patients with chronic lymphocytic leukemia, FL, and DLBCL. The shortened chair time associated with the SC formulation opens up appointment time for other patients to be scheduled. Centres can take advantage of a fixed dose and refrigerated stability to reduce drug wastage. Canadian centres that have not yet adopted the sc formulation could incorporate the lessons learned by experienced sites in terms of logistics and workflow to aid in the transition.

\section{ACKNOWLEDGMENTS}

Medical writing assistance was provided by Anna Christofides of IMPACT Medicom Inc.

\section{CONFLICT OF INTEREST DISCLOSURES}

We have read and understood Current Oncology's policy on disclosing conflicts of interest, and we declare the following interests: DS, JSA, TC, and MF have previously received honoraria from Hoffmann-La Roche Limited. EL has no conflicts of interest to disclose. Medical writing support provided by AC of IMPACT Medicom Inc. was funded by Hoffmann-La Roche Canada. AM is an employee of Hoffmann-La Roche Limited.

\section{AUTHOR AFFILIATIONS}

*Tom Baker Cancer Centre, University of Calgary, Calgary, AB; ${ }^{\dagger}$ Centre hospitalier régional de Trois-Rivières, Université de Montréal, Trois-Rivières, QC; ${ }^{\ddagger}$ The Ottawa Hospital, Ottawa, ON; ${ }^{\S}$ Burnaby Hospital, Burnaby, BC; $\|_{\text {Lymphoma Canada, Missis- }}$ sauga, ON; " IMPACT Medicom Inc., Toronto, ON; **Hoffmann-La Roche, Mississauga, ON.

\section{REFERENCES}

1. Hoffman-La Roche Ltd. Rituxan SC: Rituximab $120 \mathrm{mg} / \mathrm{mL}$ Solution for Subcutaneous Injection [product monograph]. Mississauga, ON: Hoffman-La Roche Ltd.; 2018. [Available online at: https://pdf.hres.ca/dpd_pm/00044394.PDF; cited 30 August 2019] 
2. Alberta Health Services (AHS). Lymphoma. Edmonton, AB: AHS; 2019. [Available online at: https://www.albertahealth services.ca/assets/info/hp/cancer/if-hp-cancer-guide-lyhe 002-lymphoma.pdf; cited 8 April 2020]

3. Alberta Health Services (AHS). Chronic Lymphocytic Leukemia. Edmonton, AB: AHs; 2019. [Available online at: https://www. albertahealthservices.ca/assets/info/hp/cancer/if-hp-cancerguide-lyhe007-cll.pdf; cited 30 August 2019]

4. Prica A, Baldassarre F, Hicks LK, Imrie K, Kouroukis T, Cheung M. Rituximab in lymphoma and chroniclymphocytic leukaemia: a practice guideline. Clin Oncol (R Coll Radiol) 2017;29:e13-28.

5. MacDonald D, Crosbie T, Christofides A, Assaily W, Wiernikowski J. A Canadian perspective on the subcutaneous administration of rituximab in non-Hodgkin lymphoma. Curr Oncol 2017;24:33-9.

6. Rule S, Collins GP, Samanta K. Subcutaneous vs intravenous rituximab in patients with non-Hodgkin lymphoma: a time and motion study in the United Kingdom.JMedEcon 2014;17:459-68.

7. Salar A, Avivi I, Bittner B, et al. Comparison of subcutaneous versus intravenous administration of rituximab as maintenance treatment for follicular lymphoma: results from a two-stage, phase IB study. J Clin Oncol 2014;32:1782-91.

8. Irwin S, Rowntree C, Helen C, Bloodworth C. Positive benefits of changing from intravenous rituximab administration to subcutaneous administration: a single UKcentre experience [abstract E1151]. ePoster presented at: The 21st European Hematology Association Congress; Copenhagen, Denmark; 9-12 June 2016. [Available online at: https://library.ehaweb. org/eha/2016/21st/132700/sarah.irwin.positive.benefits. of.changing.from.intravenous.rituximab.html; cited 30 April 2020]

9. Lugtenburg P, Avivi I, Berenschot H, et al. Efficacy and safety of subcutaneous and intravenous rituximab plus cyclophosphamide, doxorubicin, vincristine, and prednisone in firstline diffuse large B-cell lymphoma: the randomized MabEase study. Haematologica 2017;102:1913-22.

10. Davies A, Merli F, Mihaljevic B, et al. Pharmacokinetics and safety of subcutaneous rituximab in follicular lymphoma (SABRINA): stage 1 analysis of a randomised phase 3 study. Lancet Oncol 2014;15:343-52.
11. Rummel M, Kim TM, Aversa F, et al. Preference for subcutaneous or intravenous administration of rituximab among patients with untreated CD20+ diffuse large B-cell lymphoma or follicular lymphoma: results from a prospective, randomized, open-label, crossover study (PrefMab). Ann Oncol 2017;28:836-42.

12. Assouline S, Buccheri V, Delmer A, et al. Pharmacokinetics, safety, and efficacy of subcutaneous versus intravenous rituximab plus chemotherapy as treatment for chronic lymphocytic leukaemia (SAWYER): a phase 1b, open-label, randomised controlled non-inferiority trial. Lancet Haematol 2016;3:e128-38.

13. Hoffmann-La Roche. Roche's new time-saving subcutaneous formulation of MabThera approved in Europe for the treatment of common forms of non-Hodgkin lymphoma [media release]. Basel, Switzerland: F. Hoffmann-La Roche Ltd.; 2014. [Available online at: https://www.roche.com/media/ releases/med-cor-2014-03-28.htm; cited 24 November 2016]

14. Stewart DA, Boudreault JS, Maturi B, Boras D, Foley R. Evaluation of subcutaneous rituximab administration on Canadian systemic therapy suites. Curr Oncol 2018;25:300-6.

15. Davies AJ, Mihaljevic B, Mercadal S, et al. Longer term efficacy and safety of subcutaneous compared with intravenous rituximab: updated results of the phase 3 SABRINA study [abstract 1103]. Blood 2016;128:.

16. Hematology Times. Health Canada approves SC rituximab for CLL [online news article]. Parsippany, NJ: Frontline Medical Communications Inc.; 2018. [Available at: https:// www.mdedge.com/hematology-oncology/article/185060/ leukemia-myelodysplasia-transplantation/health-canada -approves-sc-rituximab; cited 30 August 2019]

17. Institut national d'excellence en santé et en services sociaux. Rituxan et Rituxan SC-Lymphome [French]. Quebec City, QC: Gouvernement du Québec; 2019. [Available online at: https://www.inesss.qc.ca/fileadmin/doc/INESSS/ Inscription_medicaments/Avis_au_ministre/Mai_2019/ Rituxan_2019_05.pdf; cited 12 August 2019]

18. Favier M, Le Goc-Sager F, Vincent-Cantini I, et al. Medicoeconomic benefits of subcutaneous formulations of trastuzumab and rituximab in day hospitalisation (scuBA study) [French]. Bull Cancer 2018;105:862-72. 